\title{
¿Qué hacen los extranjeros en Medellín? Grupo Focal
}
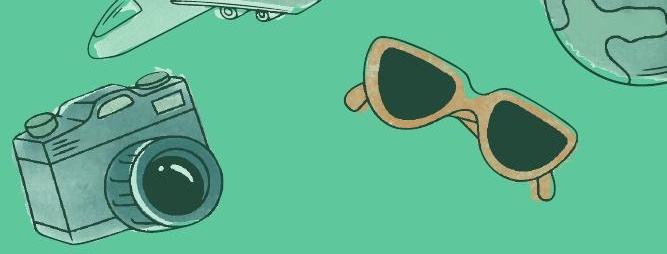

* Evelyn Sáez; Valeria Montes de Oca Ramírez; Manuela Ardila Diosa;María Jessenia Pabón Buitrago;

Valentina López Álvarez; Eva Sandrith Machado Montoya; Sergio Iván Barriga Maldonado; Laura Álvarez Saldarriaga; Alejandro Cadavid Cadavid; Valentina Ospina Barco; Diana Isabel Álvarez Giraldo; Jhoana Carolina Acevedo;
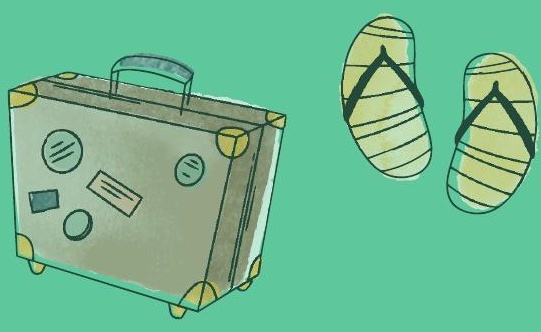
Andrea Lopera Ceballos

DOI: https://doi.org/ 10.33571/revinterseccion.v2n4a3

\section{Resumen}

El artículo presenta el proceso y resultado del grupo focal adelantado para generar información en torno a las experiencias de los extranjeros en la ciudad de Medellín. La técnica fue aplicada por estudiantes de la Tecnología en Producción de Eventos del Politécnico Colombiano Jaime Isaza Cadavid, en el Seminario de investigación aplicada.

Se encuentra que los extranjeros llegaron a Medellín por intercambio académico, también en busca de oportunidades para crear empresa, vacaciones, trabajo y por romance. A todos le gusta viajar a los municipios cercanos y tener aventuras en la ciudad. El evento que más recuerdan es el de la Feria de la Flores; les gusta asistir a eventos deportivos como el fútbol y el básquetbol, también asisten a fiestas en bares. Les gustaría más programación de actividades urbanas culturales al aire libre.

Palabras claves: Medellín; extranjeros; producción de eventos; grupo focal.

Recibido. Agosto 19, 2020

Aceptado. Septiembre 20,2020

*Estudiantes del curso seminario de investigación aplicada de la Tecnología en Organización de Eventos del Politécnico Colombiano Jaime Isaza Cadavid coordinado por la profesora Mónica Valle; email: mmvalle@elpoli.edu.co ; Estudiantes redactoras: evelyn_saez35181@elpoli.edu.co ; manuela_ardila35181@elpoli.edu.co ; valeria_ montes35181@elpoli.edu.co 


\section{What do foreigners do in Medellín? Focus Group}

* Evelyn Sáez; Valeria Montes de Oca Ramírez; Manuela Ardila Diosa;María Jessenia Pabón Buitrago; Valentina López Álvarez; Eva Sandrith Machado Montoya; Sergio Iván Barriga Maldonado; Laura Álvarez Saldarriaga; Alejandro Cadavid Cadavid; Valentina Ospina Barco; Diana Isabel Álvarez Giraldo; Jhoana Carolina Acevedo; Andrea Lopera Ceballos

DOI: hitps://doi.org/ 10.33571/revinterseccion.v2n4a3

\section{Abstract}

The article introduces the process and results of the focus group conducted to generate information about the experiences of foreigners in Medellin city. The technique was conducted by students of the Technology in Event Production of the Politécnico Colombiano Jaime Isaza Cadavid, in the Seminar of applied research.

The findings show foreigners come to Medellin for academic exchange, also they look for business opportunities, vacations, work, and romance. They all like to travel to nearby municipalities and enjoy adventures in the city. The event they remember the most is the Flower Fair; in addition, they like to attend sporting events such as soccer and basketball, they also attend parties in bars. They would like more offering of outdoor urban cultural activities.

Keywords: Medellin; foreigners; event production; focus group.

\section{Received. August 19, 2020 Accepted. September 20, 2020}

*Students of the applied research seminarcourse of the Technology in Event Organization of Politécnico Colombiano Jaime Isaza Cadavid coordinated by Professor Mónica Valle; email: mmvalle@elpoli.edu.co. Writing students: evelyn_saez35181@elpoli.edu. co; manuela_ardila35181@elpoli.edu.co ; valeria_montes35181@elpoli.edu.co. 


\section{¿O que é que os estrangeiros fazem em Medellín? Grupo de foco}
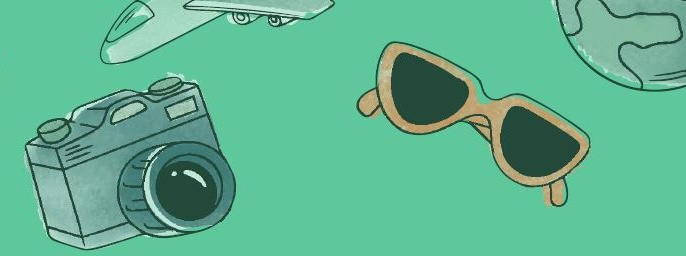

* Evelyn Sáez; Valeria Montes de Oca Ramírez;

Manuela Ardila Diosa;María Jessenia Pabón Buitrago;

Valentina López Álvarez; Eva Sandrith Machado Montoya;

Sergio Iván Barriga Maldonado; Laura Álvarez Saldarriaga;

Alejandro Cadavid Cadavid; Valentina Ospina Barco;

Diana Isabel Álvarez Giraldo; Jhoana Carolina Acevedo;

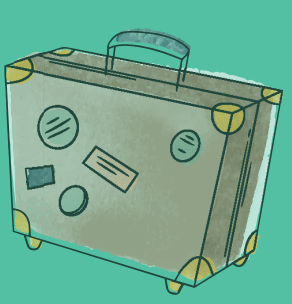

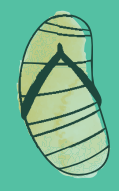

Andrea Lopera Ceballos

DOI: https://doi.org/ 10.33571/revinterseccion.v2n4a3

\section{Resumo}

O artigo apresenta o processo e os resultados do grupo focal realizado para gerar informação sobre as experiências de estrangeiros na cidade de Medellín. A técnica foi aplicada por estudantes da Tecnologia em Produção de Eventos do Politécnico Colombiano Jaime Isaza Cadavid, no Seminário de investigação aplicada.

Descobriu-se que os estrangeiros vieram a Medellín para intercâmbio académico, também em busca de oportunidades de negócios, férias, trabalho e romance. Todos eles gostam de viajar para municípios próximos e ter aventuras na cidade. O evento de que mais se lembram é a Feira das Flores; gostam de assistir a eventos esportivos como o futebol e o basquetebol, e também assistem a festas em bares. Gostariam de ter mais programação para atividades culturais urbanas ao ar livre.

Palavras chave: Medellín; estrangeiros; produção de eventos; grupo focal.

Recebido. Agosto 19, 2020

Aceitado. Setembro 20, 2020

* Estudantes do seminário de investigação aplicada do curso de Organização de Tecnologia em Eventos do Politécnico Colombiano Jaime Isaza Cadavid coordenado pela Professora Mónica Valle; email: mmvalle@elpoli.edu.co ; Editores Estudantes: evelyn_saez35181@elpoli.edu.co ; manuela_ardila35181@elpoli.edu.co ; valeria_montes35181@elpoli.edu.co 


\section{Autoras(es)}

Evelyn Sáez; Valeria Montes de Oca Ramírez; Manuela Ardila Diosa; María Jessenia Pabón Buitrago; Valentina Lopez Alvarez; Eva Sandrith Machado Montoya; Sergio (lak Bariga Maldonado; Laura Alvarez Saldarriaga; Din Andrea Lopera Ceballos.

\section{¿Qué hacen los extranjeros en Medellín? Grupo Focal.}

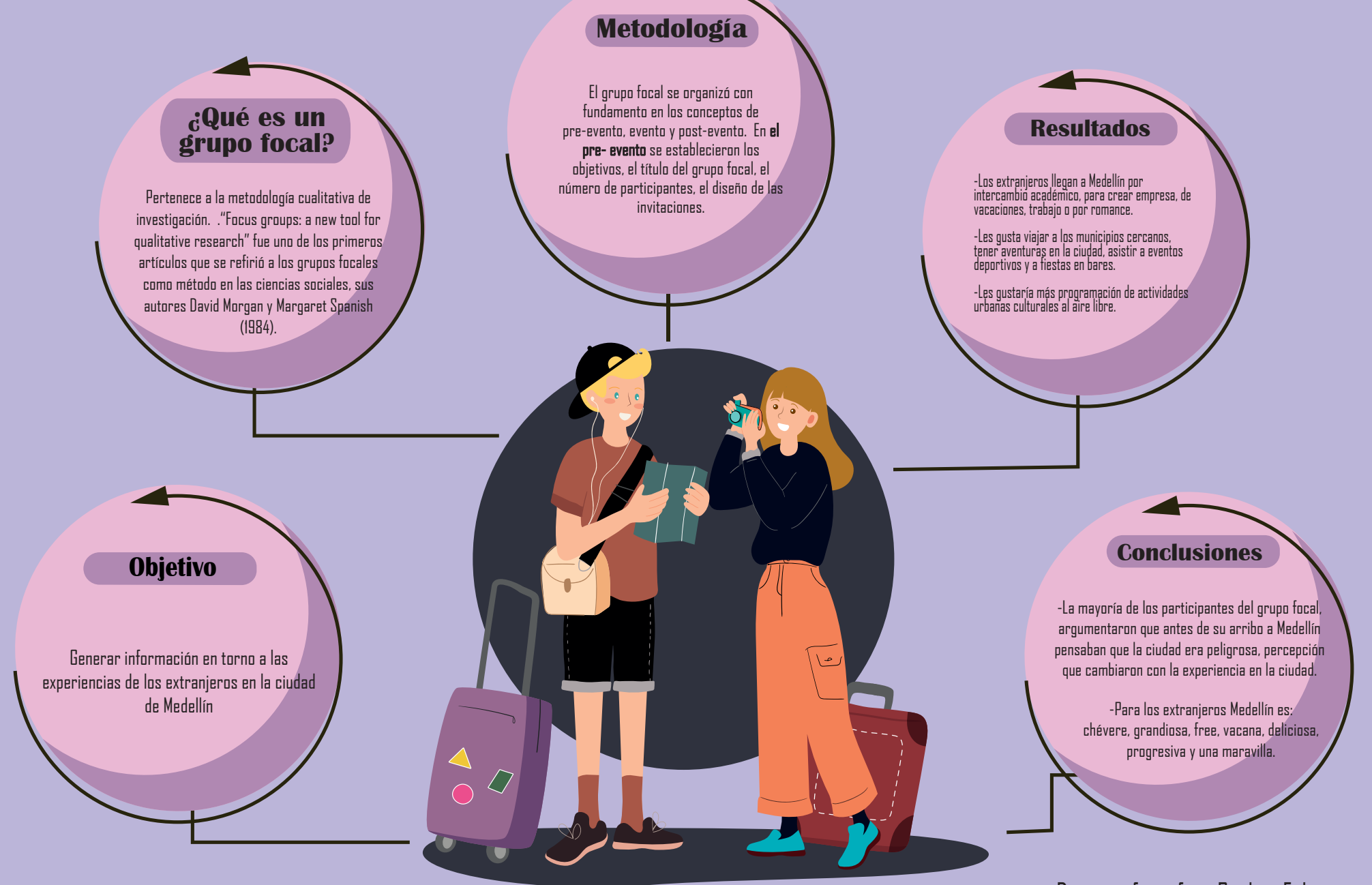

Diseño infográfica: Paulina Echeverri 


\section{Introducción}

- conocimiento de los públicos, sus intereses y - necesidades se constituye en información relevante para que los productores de eventos diseñen propuestas que sean pertinentes para éstos. En tal sentido se realizó un grupo focal con extranjeros residentes y visitantes de Medellín.

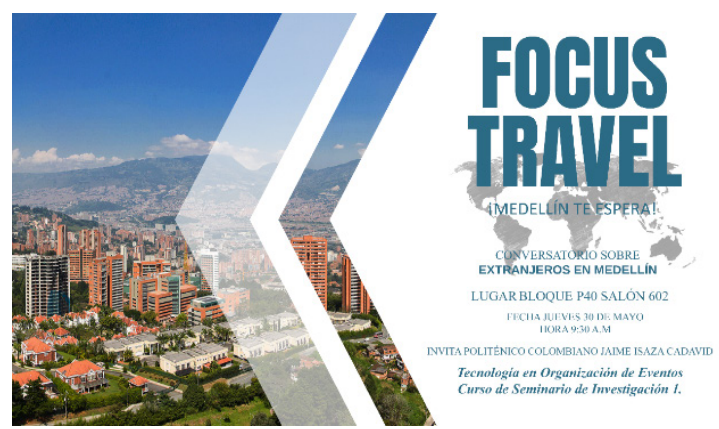

Elaboración propia. Invitación a la actividad en inglés y en español

El grupo focal tuvo como objetivos conocer las razones de la estancia de los extranjeros en la ciudad así como algunos de sus datos sociodemográficos (nombre, edad, lugar de origen, ocupación), eventos a los que había asistido en la ciudad y los que les gustaría se realizaran; las interacciones que sostienen durante su permanencia en la ciudad; percepción de Medellín antes de llegar y luego de vivir la experiencia; diferencias y semejanzas entre el lugar de origen del extranjero y Medellín. También se les solicitó describir, en una palabra, la ciudad.

Los extranjeros que participaron del grupo focal fueron: Sybylla Jockyman do Canto Serafini (Brasil); Daniel Otavio Vinques Serafini (Brasil); Thomas Lusser (Suecia); Tom Soleillant (Francia); Lucia Gavidia (Perú); Mathew Rideout (USA); Nickolas Saraceni (USA); Calvin Smith (USA); Juliet (Holanda) y Patricia Esponda (México).

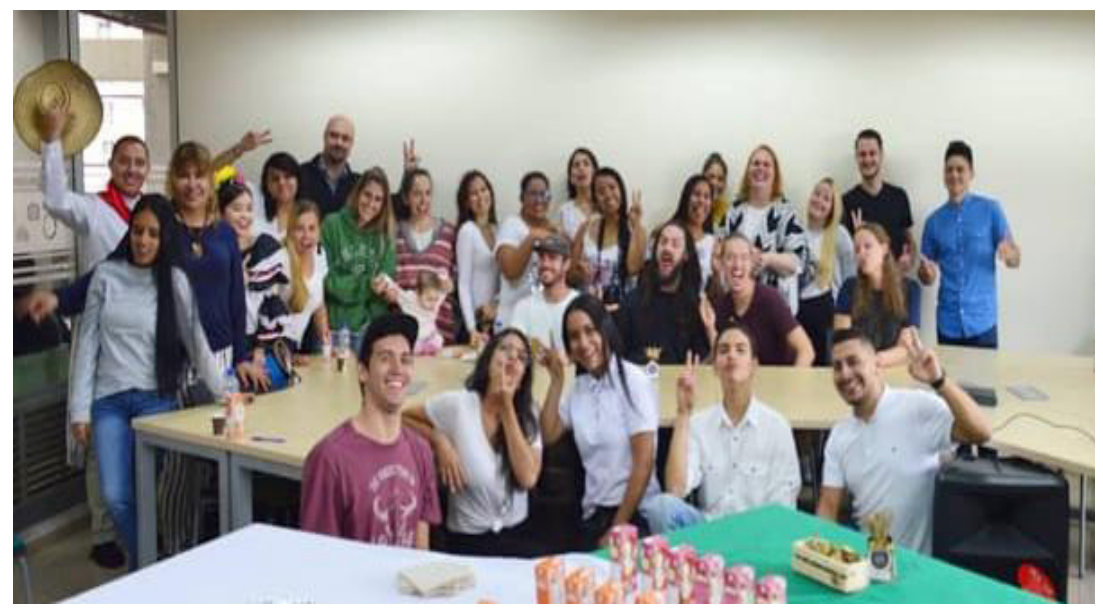


Para la realización del conversatorio se designó un moderador quien contextualizó a los participantes en este evento académico. Les informó de la temática y la dinámica a desarrollar. Les anunció que contaban con traductor para quienes quisieran hablar en su idioma original. La actividad se desarrolló mediante un dialogo fluido entre los participantes, lo que se logró gracias a los organizadores que generaron confianza. Al final del evento se compartieron empanadas que son "pasabocas" tradicional de Colombia también el café y otros aperitivos. Se obsequió un souvenir que consistió en un dulce de coco y una manilla de Medellín, esto como estrategia para generar recordatorio de su participación en el grupo focal y también de Medellín como ciudad hospitalaria. Una gran sorpresa para los participantes fue la presentación de una pareja de baile que danzaron al ritmo de la música tradicional de país.
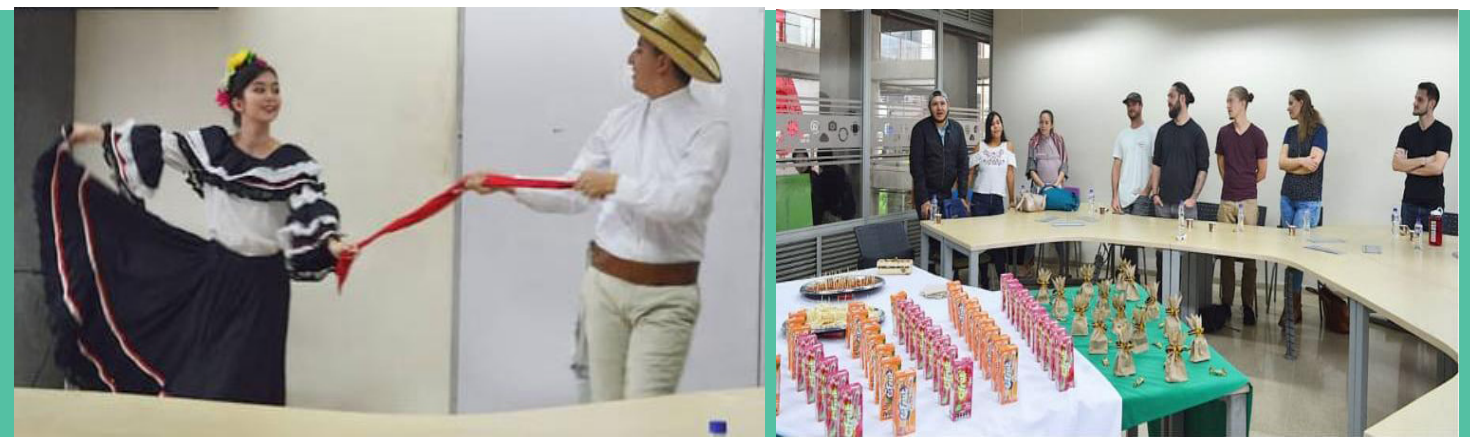

En este artículo se presentan los resultados de este grupo focal, se describe como se organizó y se reflexiona en torno a esta técnica de la investigación cualitativa para la obtención de datos.

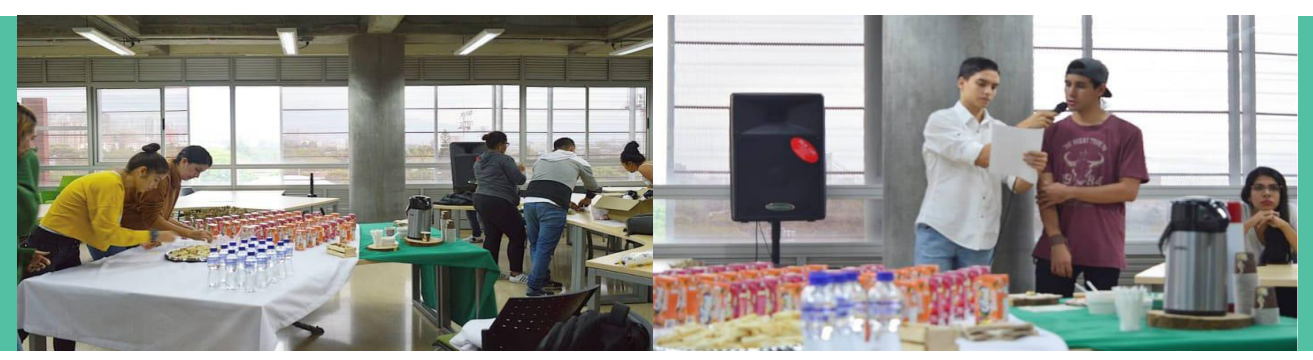

\section{1. ¿Qué es un grupo focal?}

El grupo focal es una técnica (Olson,2000) utilizada para la generación de información en relación a un determinado fenómeno o tema. Pertenece a la metodología cualitativa de investigación esto es aquella que no depende de medios de cuantificación o procedimientos estadísticos para encontrar hallazgos. Este tipo de investigación permite describir un grupo o una cultura. Utiliza otras técnicas como la observación participante, la entrevista, los informantes claves, las biografías, entre otros.

La labor del científico es entrevistar a personas; hace consideraciones de la opinión de persona sobre la opinión de otra, busca lazos comunes así como diferentes. Estudia asuntos cotidianos, patrones de comportamiento y del pensamiento que ocurre día a día.

Las ciencias sociales han utilizado los grupos focales desde mediados de los años 20, R.Merton y P.Lazarsfeld realizaron entrevistas grupales antes de la Segunda Guerra Mundial. Ellos aplicaron esta técnica para crear manuales de entrenamiento para las tropas, también para analizar la segregación racial al interior de dichas tropas. Después de la guerra, Merton y dos de sus estudiantes escribieron un libro considerado clásico sobre grupos focales, "The focused interview" 
Entre 1950 y 1980 se les conoció como "group depth interviews" su énfasis en la profundidad se debía al interés en descubrir las motivaciones psicológicas de los consumidores. "Focus groups: a new tool for qualitative research" fue uno de los primeros artículos que se refirió a los grupos focales como método en las ciencias sociales, sus autores David Morgan y Margaret Spanish (1984). En los años 90, Michael Patton y Richard Krueger los utilizaron en la educación para evaluar sus problemáticas.

En comparación con otras técnicas el grupo focal es relativamente de bajo costo, permite la obtención rápida de resultados. Esta técnica es usual en estudios de mercadeo y publicidad. Un grupo focal típico está compuesto entre 6 a 10 participantes. Una sesión debiera durar mínimo 90 y máximo 120 minutos. Uno de los costos de un grupo focal son los incentivos que se deben dar a los participantes en tanto que participar en un grupo focal demanda tiempo y probablemente gastos de traslado. Por ello, es natural tratar de dar un incentivo material a las personas que participan.

"Cuando se planifica el grupo focal y se sabe con exactitud lo que se desea obtener de su realización, la perspectiva estructurada es la mejor alternativa. En cambio, grupos focales menos estructurados son útiles para propósitos exploratorios. Escuchar aquellos tópicos elegidos por los participantes como temas de discusión en un grupo menos estructurado ayudará a obtener sus propias perspectivas sobre el tema general que interesa al grupo focal. La desventaja en todo caso es que el grupo puede ser un tanto errático en términos de su productividad" Mella (2000).

El formato de la discusión la ofrece el moderador o facilitador quien es flexible para explorar asuntos que no hayan sido anticipados.

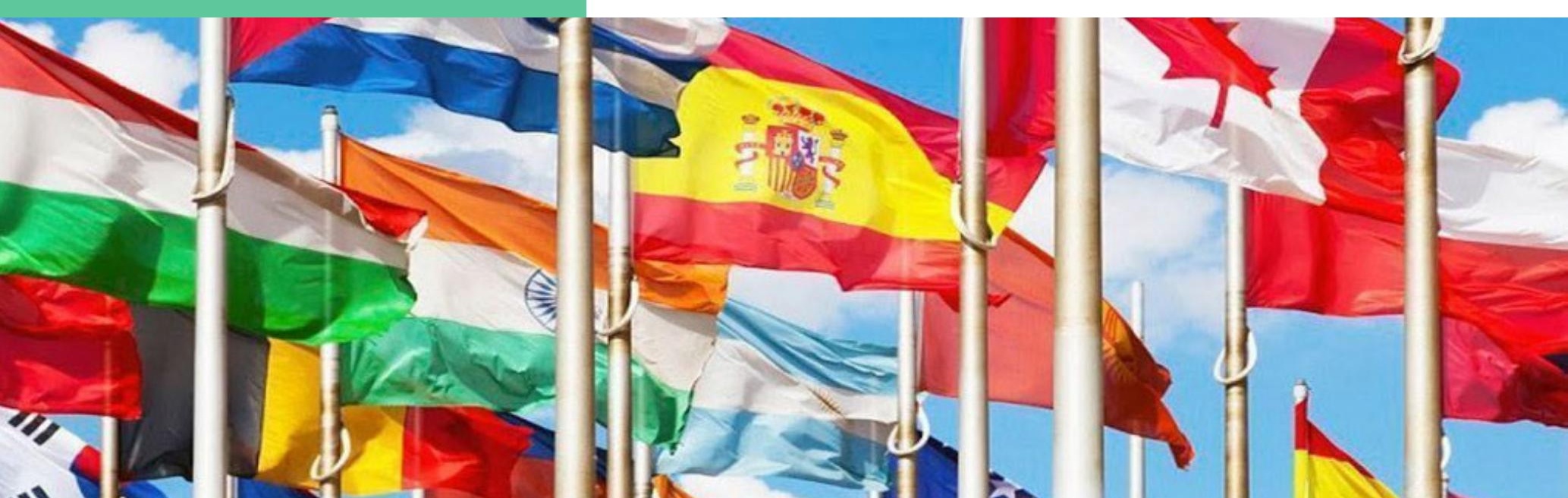




\section{Metodología}

El grupo focal se organizó con fundamento en los conceptos de pre-evento, evento y postevento. En el pre- evento se establecieron los objetivos, el título del grupo focal, el número de participantes, el diseño de las invitaciones. Los organizadores se distribuyeron funciones y crearon comités responsables de la ejecución de éstas.

Comités: Coordinación: Valentina Ospina. RR. PP: Valeria Montes de Oca y Manuela Ardila. Logística: Laura Álvarez, Valentina López Y Andrea Lopera. Fotografía: Eva Machado y Jhoana Acevedo. Presupuesto: Alejandro Cadavid. Alimentación: Diana Álvarez. Comunicación e Imagen: Sergio Barriga y Jessenia Pabón. Redacción: Evelyn Sáez, Manuela Ardila y Valeria Montes de Oca.

Se determinó la fecha y hora del evento, el lugar; se estableció el cronograma general de la actividad, se determinaron los recursos humanos, materiales, técnicos y financieros requeridos para la realización de éste.

\section{Tabla. 1. Cronograma de actividades}

\begin{tabular}{|c|c|c|c|c|c|}
\hline \multirow{2}{*}{ ACTIVIDAD } & \multicolumn{2}{|r|}{ MAYO } & \multirow{2}{*}{ RESPONSABLES } & \multirow{2}{*}{ HORARIO } & \multirow{2}{*}{ OBSERVACIONES } \\
\hline & 27 & \begin{tabular}{l|l|l}
28 & 29 & 3 \\
\end{tabular} & & & \\
\hline \multicolumn{6}{|c|}{ Pre-evento } \\
\hline Entrega de cuota presupuestal & $x$ & & Comité de presupuestos & No aplica & Alejandro Cadavid \\
\hline $\begin{array}{l}\text { Confirmación de extranjeros e } \\
\text { Invitación a extranjeros restantes }\end{array}$ & $x$ & & Todos los integrantes & No aplica & $\begin{array}{l}\text { Ir a los lugares recomendados } \\
\text { para contactar extranjeros }\end{array}$ \\
\hline $\begin{array}{l}\text { Gestionar y confirmar barra de } \\
\text { Sonido }\end{array}$ & $x$ & & Comité de logistica & No aplica & Con Diana Barreneche \\
\hline $\begin{array}{l}\text { Solicitary confirmar manteles } \\
\text { blancos }\end{array}$ & & $x$ & Comité de logistica. & No aplica & Con Santiago Correa \\
\hline Resenvar cámaras fotográficas & & $x$ & Comité de comunicaciones & No aplica & $\begin{array}{c}\text { Enfatizar en la carga de la batería } \\
\text { de las cámaras }\end{array}$ \\
\hline
\end{tabular}

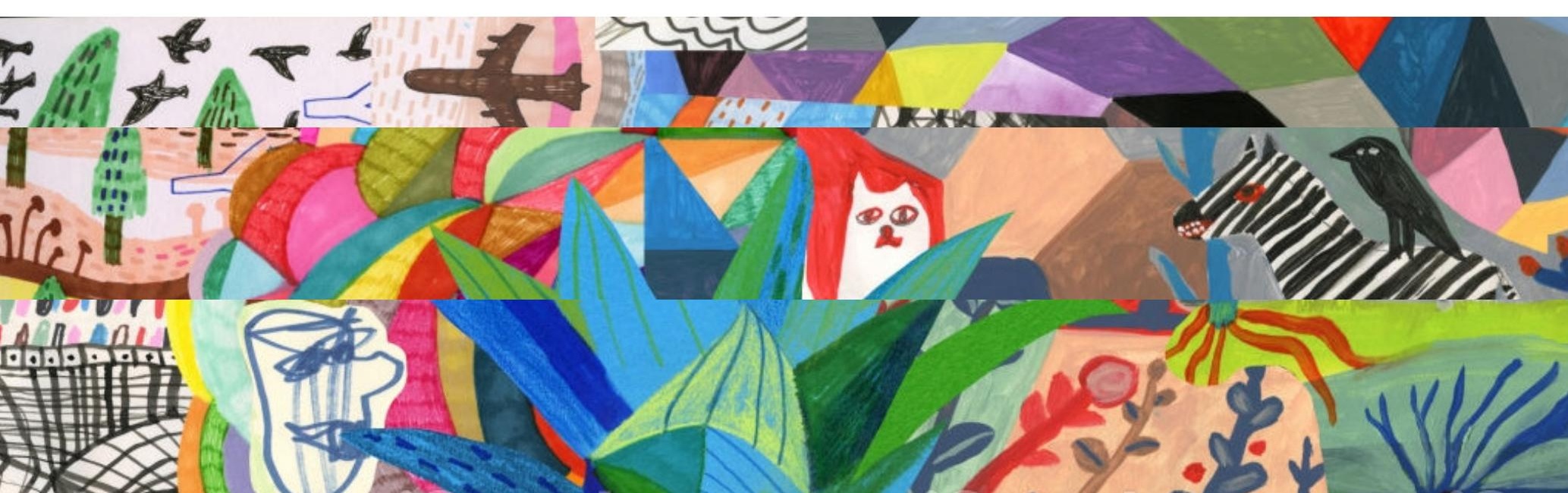




\begin{tabular}{|c|c|c|c|c|}
\hline \multicolumn{5}{|c|}{ Evento } \\
\hline Inicio de montaje & $x$ & Todos los integrantes & 7:45 a. m. & $\begin{array}{l}\text { Contar con el salón en } \\
\text { disposición y llegar con todos los } \\
\text { insumos asignados a cada } \\
\text { comité y a cada participante }\end{array}$ \\
\hline Disponer el mobiliario & $x$ & Todos los integrantes & 8:00 a. m. & $\begin{array}{c}\text { Ir por la barra de sonido, cámaras } \\
\text { y manteles. }\end{array}$ \\
\hline $\begin{array}{c}\text { Disponer de la estación de } \\
\text { comida y bebidas }\end{array}$ & $\mathrm{x}$ & Comité de alimentación & 9:00 a. m. & \\
\hline $\begin{array}{c}\text { Disponer los videos en la } \\
\text { nantalla }\end{array}$ & $x$ & Comité de logística y fotografia & 9:00 a. m. & Verificar sonido \\
\hline $\begin{array}{l}\text { Estar en la entrada del poli, por el } \\
\text { metro, para recibir a los invitados }\end{array}$ & $x$ & Valeria, Sergio, Jesenia, Valentina López & 9:15 a. m. & $\begin{array}{c}\text { Ser muy amables y guarlos hasta } \\
\text { el salón correspondiente. Al } \\
\text { traerlos, devolverse a la portería } \\
\text { nuevamente }\end{array}$ \\
\hline Estar en posiciones & $x$ & Todos los integrantes & 9:15 a. m. & $\begin{array}{c}\text { Cuidar de que ya esté disponible } \\
\text { el café para servir a los que van } \\
\text { entrando }\end{array}$ \\
\hline $\begin{array}{c}\text { Ofrecer café y agua al ingreso de } \\
\text { los invitados } \\
\end{array}$ & $x$ & Comité de alimentación & 9:15 a.m. & $\begin{array}{c}\text { Solamente se ofrecen bebidas } \\
\text { hasta las } 9: 30\end{array}$ \\
\hline $\begin{array}{c}\text { Comenzar a proyectar los videos } \\
\text { en la pantalla } \\
\end{array}$ & $x$ & Comité de comunicaciones & 9:15 a. m. & \\
\hline Proyección de videos & $\mathrm{x}$ & Comité de comunicaciones & 9:15 a.m. - 9:35 a. m. & \\
\hline (inicio del evento) Bienvenida & $\mathrm{x}$ & Alejandro & 9:35:00 a. m. - - $: 38$ a. m. & Cuenta con 3 minutos \\
\hline Rompe hielo & $\mathrm{x}$ & Valentina Ospina & 9:38:00 a. m. - 9:53 a. m. & Cuenta con 15 minutos \\
\hline Introducción a las preguntas & $\mathrm{x}$ & Alejandro & 9:53 a. m. - 9:55 a. m. & Cuenta con 2 minutos \\
\hline Conversatorio & $\mathrm{x}$ & Moderador: Alejandro & 9.55 a. m. - 10.55 a. m. & 1 hora \\
\hline $\begin{array}{l}\text { Instalar la pista de la muestra } \\
\text { cultural en la barra de sonido }\end{array}$ & $x$ & Valentina Ospina & 9:55 a. m. & \\
\hline Muestra cultural & $\mathrm{x}$ & Valentina Ospina & 10:55 a. m. - 11:05 a. m. & \\
\hline Entrega de pasabocas & $\mathrm{x}$ & Comité de alimentación & 10:55 a. m. - 11:05 a. m. & \\
\hline Despedida & $\mathrm{x}$ & Alejandro & 11:05 a. m. a 11:10 a. m. & Cuenta con 5 minutos \\
\hline $\begin{array}{c}\text { Estar en la entrada del salón para } \\
\text { entregar los souvenir }\end{array}$ & $x$ & Jesenia, Valentina López & 11:10 a. m. - 11:15 a. m. & \\
\hline $\begin{array}{l}\text { Acompañar a la salida a los } \\
\text { extranjeros que así requieran }\end{array}$ & $x$ & Valeria, Sergio & $11: 15$ a. m. & \\
\hline
\end{tabular}

El evento fue la actividad propiamente dicha de la cual se da cuenta en este artículo. En el post- evento, se procedió a organizar el salón utilizado para la actividad para su entrega; se compiló el material audiovisual, notas de campo y fotográfico con el que se registró la sesión. Se procedió a brindar los agradecimientos respectivos, se evalúo la actividad en tantos los aspectos positivos y por mejorar. Los organizadores también analizaron el cumplimiento de los compromisos y funciones.

\section{Resultado}

Este grupo focal se inicia con una presentación en el que el maestro de ceremonia y el traductor, explican la mecánica del grupo focal, luego se procede a romper el hielo entre los participantes con una actividad lúdica.

Se da inició oficial al grupo focal, un joven Suizo de 26 años, comenta que él se enamoró de Medellín desde el 2013 cuando realizó un viaje por Latinoamérica, a raíz de esto visitó la ciudad cada año, realizó un intercambio académico en la ciudad y decidió radicarse en este lugar y abrir una empresa de marketing y mercadeo. Antes de visitar la ciudad la percepción que tenía de Medellín era lo que se mostraba en la Tv y las películas en las que se veía que la ciudad era peligrosa, cuando visitaba otros países y ciudades de la región también le decían que no viniera a Medellín porque corría muchos riesgos.

Su opinión sobre la ciudad cambio totalmente ahora que ha estado tanto tiempo en la ciudad, le gusta visitar municipios cercanos a Medellín como San Antonio de Prado y San Cristóbal, generalmente viaja en su moto. En cuanto a las semejanzas de Medellín con su lugar de origen señala que en Suiza al igual que en Colombia se come mucho chocolate con queso. Entre los eventos que ha asistido está la Feria de las Flores. 


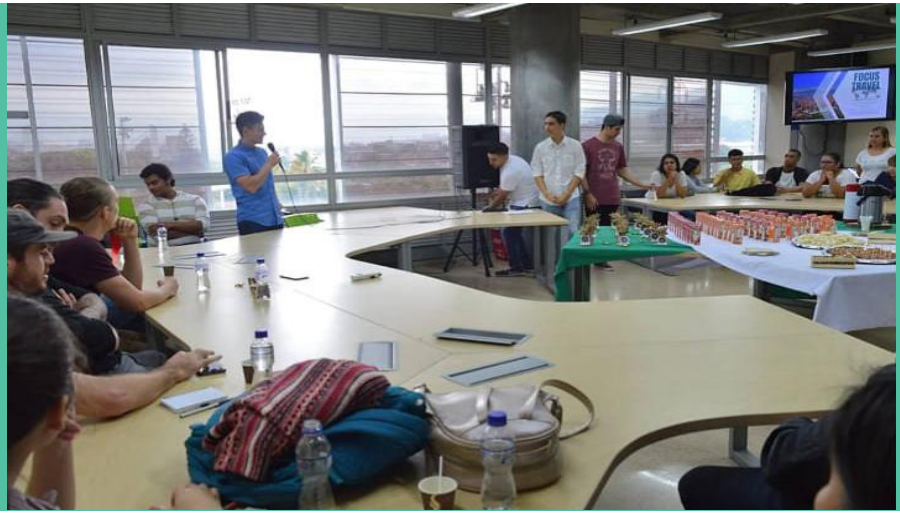

Otro de los participantes comento que se vino a Medellín porque se cansó de vivir en Francia, le aburrió la cotidianidad de ese país. Desde su arribo se propuso descubrir la ciudad, ha viajado en metrocable, le gusta recorrer los barrios y ver el urbanismo. Entre los eventos a los que ha asistido están los partidos de fútbol, fiestas en bares. Comentó que el idioma francés y el español son muy parecidos porque ambos provienen del Latín.

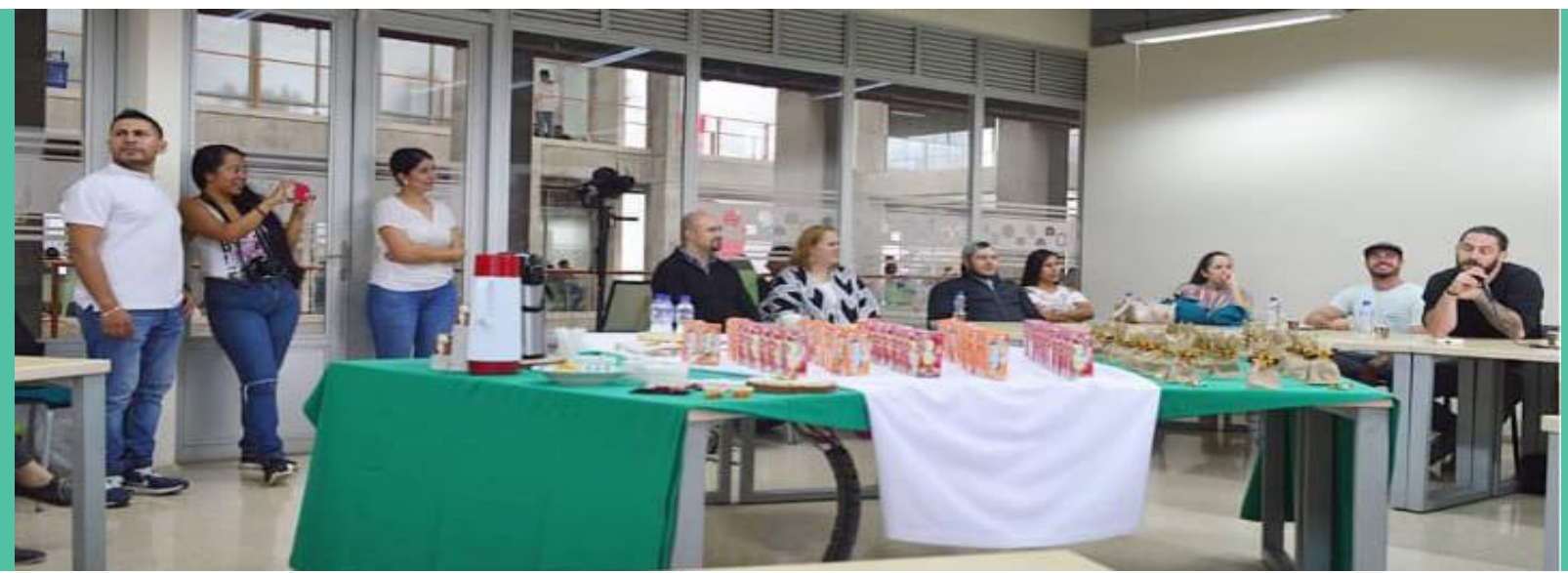

La participante de Holanda mencionó que ella contaba con tres semanas de vacaciones y se vino a conocer estos lares, primero llegó a Cuba, después viajó a Medellín pensaba que esta ciudad era muy peligrosa, sólo sabía de Pablo Escobar y de narcos pero toda Colombia la sorprendió. En Medellín ha asistido a eventos de intercambio de idiomas, eventos culturales. Para ella su país y Colombia o Medellín no tienen similitudes. Le gustaría que se realizaran eventos en los que pudiera conocer a otros extranjeros que visitan la ciudad.

El extranjero oriundo de Estados Unidos, indicó que tenía 29 años que provenía de Denver. Él estuvo en Brasil antes de arribar a Medellín, expresa que no le gustó el idioma portugués, por eso y porque se enteró del premio que recibió Medellín como ciudad emprendedora decidió venir a esta ciudad. Aquí trabaja en el SENA enseñando inglés. Le encanta esta ciudad por su cultura, la gente emprendedora y acogedora. Dice que en Medellín se siente como si estuviera en Denver ya que hay muchas montañas. De este lugar le agrada la amabilidad de la gente. Él asiste frecuentemente a encuentros de básquetbol. Como se encontró con tres amigos en la ciudad, están conformando una empresa de tecnología y marketing digital.

El otro americano, amigo y socio del anterior vivió en Japón y de allí recorrió Suramérica y ahora está radicado en Medellín. Él y su socio asisten a eventos de rap y también les gustan en los que se intercambian las culturas. Este participante del grupo focal comenta que en California de donde es oriundo el arte urbano es casi igual al de acá. El tercer americano comenta que trabajó en Google en diferentes proyectos y que su plan era ir a México para aprender el idioma español pero terminó en Medellín, a él no le gusta la rutina. Su hobby es tomar fotos, realizar videos. Le gustan los graffiti y la actividad cultural urbana. Ha asistido a eventos como pintar murales y a actividades lúdicas al aire libre. Él encuentra como similitud entre Medellín y su lugar de origen que las personas colaboran. Expresó que "los gringos" piensan que Medellín es peligroso. 
Después de la alocución liderada por los hombres, una joven mexicana tomó la palabra comentó que está en el País porque vino de intercambio estudiantil a la universidad Santo Tomás en Bogotá, se conoció con su esposo allí y se casó, como al esposo lo trasladaron a Medellín se vinieron para acá. Ella está más feliz en esta ciudad ya que en Bogotá hace mucho fio. Ella pensaba que los antioqueños eran presumidos como los argentinos pero se ha dado cuenta de que no es así por el contrario encuentra una cultura que es muy colectiva y amigable. Ella ha asistido a eventos religiosos, eventos de niños, y eventos de fines de semana. Encuentra como similitud entre su cultura y la de Medellín la música, también que se comen frijoles, la práctica del deporte y la religiosidad de la gente.

La participante peruana compartió que también había llegado a la ciudad por intercambio estudiantil a la Universidad CES. Lo que ella sabía de la ciudad antes de llegar era lo que veía en Netflix, películas de narcos. Ahora piensa que las personas son amables. Ella asiste a partidos de fútbol. Le parece que Perú se parece a Colombia por el frío de sus ciudades capitales.

Los últimos extranjeros que compartieron su experiencia fue una pareja de Brasil, ella enseña portugués a extranjeros, también estudia un doctorado, al esposo le gusta Medellín por el clima.

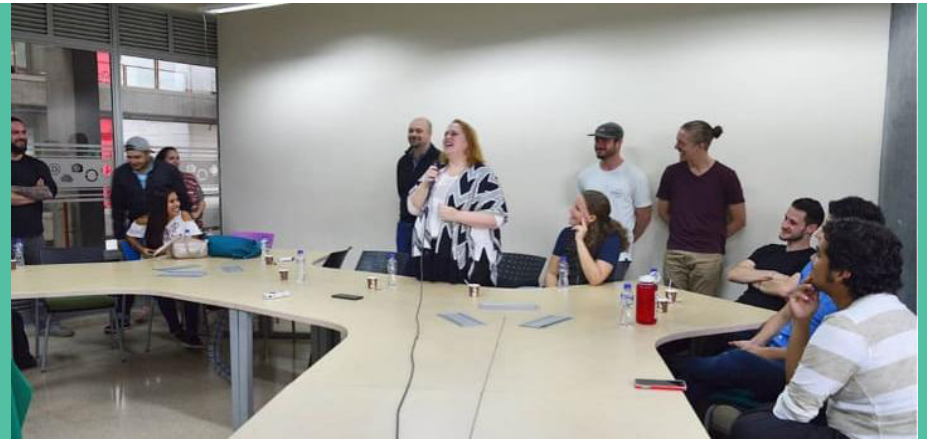

"Brasil tiene los mismos problemas que Medellín, todos los países tienen problemas. Es necesario que se exporte lo bueno y no lo malo".

Ellos han asistido a eventos como el de las Feria de las Flores. Ella fue la traductora para la gobernación de Antioquia en el trágico accidente del avión del Chapecoense. Para ellos Medellín y Brasil tienen más similitudes que diferencias, por ejemplo a su gente les gusta el café, el fútbol, el "Romeo y Julieta" que es el queso con bocadillos, también el aguacate, "aunque en el Brasil hacen dulce con esto y aquí se come con sal".

Finalmente un asistente que hospedaba extranjeros participó en el grupo focal y comentó que los alemanes y los hindú preparaban recetas extrañas que ni siquiera se encuentran esos ingredientes en Medellín.

\section{A modo de conclusión}

La mayoría de los extranjeros que participaron en el grupo focal argumentaron que antes de su arribo a Medellín pensaban que la ciudad era peligrosa, percepción que cambiaron con la experiencia en la ciudad. De este lugar les gusta el clima, las montañas, los paisajes, sus habitantes, el emprendimiento y las ganas de salir adelante. Describieron esta ciudad como: felicidad, chévere, grandiosa, free, vacana, deliciosa, progresiva, una maravilla.

Se encuentra que los extranjeros llegaron a Medellín por intercambio académico, también en busca de oportunidades de hacer empresa, vacaciones o por trabajo. A todos le gusta viajar a los municipios cercanos y tener aventuras en la ciudad. El evento que más recuerdan es el 
de la Feria de la Flores; les gusta asistir a eventos deportivos como el fútbol y el básquetbol, también asisten a fiestas en bares. Les gustaría más programación de actividades urbanas culturales al aire libre.

Los participantes del grupo focal se catalogan como jóvenes, sus edades estaban entre los 26 y 45 años. Todos contaban con alto nivel de estudios, tienen una profesión y están interesado en aportar a la ciudad. La mayoría encuentran referentes entre su país de origen y la ciudad, entre estos algunos alimentos como el chocolate y el queso, el gusto por el café y el aguacate.

La aplicación del grupo focal permite la generación de información desde diferentes puntos de vista según el grupo convocado. Con esta actividad se logaron los objetivos planteados, se aprendió con fundamento en la experiencia cómo programar y ejecutar un grupo focal, también se fortaleció el trabajo en equipo, se participó de un intercambio cultural. Esta actividad nos motivó a realizar más estudios sobre este tema.

\section{Bibliografía}

Reyes, T. (1999). Métodos cualitativos de investigación: los grupos focales y el estudio de caso. Fórum Empresarial, , Vol. 4, №. 1, 1999, págs. 74-87 . Disponible en: https://dialnet.unirioja.es/ servlet/articulo? codigo $=6230192$

Mella, O. ( 2000). Grupos Focales ("Focus Groups"). Técnica de investigación cualitativa. Publicado como Documento de Trabajo N 3, CIDE, Santiago, Chile. Disponible en:

iles.palenque-de-egoya.webnode.es/200000285- 1 b8502a79/Grupos\%20Focales\%20de\%20 Investigación.pdf

Valle, M.; Marín, E. (2015). Etnografía de la teleaudiencia de Medellín. Hábitos, uso y contextos de consumo. Luciérnaga Comunicación, 6(11), 1-14. https://doi.org/10.33571/ revistaluciernaga.v6n 1 la 1

Para citar este artículo

Sáez, E.; Montes de Oca, V.; Ardila, M., \& Otros (2021). ¿Qué hacen los extranjeros en Medellín? Grupo focal. Revista Intersección: Eventos, turismo, moda y gastronomía. Vol. 2 Núm. 4. Pp: 37-48; https://doi.org/ 10.33571/revinterseccion.v2n4a3

OJS: https://revistas.elpoli.edu.co/index.php/int/issue/archive Link: https://www.politecnicojic.edu.co/index.php/revista-interseccion 